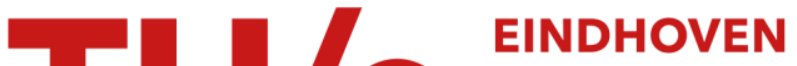 \\ UNIVERSITY OF \\ TECHNOLOGY
}

\section{Clustering-structure representative sampling from graph streams}

Citation for published version (APA):

Zhang, J., Zhu, K., Pei, Y., Fletcher, G. H. L., \& Pechenizkiy, M. (2017). Clustering-structure representative sampling from graph streams.

Document status and date:

Published: 01/01/2017

\section{Document Version:}

Typeset version in publisher's lay-out, without final page, issue and volume numbers

\section{Please check the document version of this publication:}

- A submitted manuscript is the version of the article upon submission and before peer-review. There can be important differences between the submitted version and the official published version of record. People interested in the research are advised to contact the author for the final version of the publication, or visit the $\mathrm{DOI}$ to the publisher's website.

- The final author version and the galley proof are versions of the publication after peer review.

- The final published version features the final layout of the paper including the volume, issue and page numbers.

Link to publication

\section{General rights}

Copyright and moral rights for the publications made accessible in the public portal are retained by the authors and/or other copyright owners and it is a condition of accessing publications that users recognise and abide by the legal requirements associated with these rights.

- Users may download and print one copy of any publication from the public portal for the purpose of private study or research.

- You may not further distribute the material or use it for any profit-making activity or commercial gain

- You may freely distribute the URL identifying the publication in the public portal.

If the publication is distributed under the terms of Article $25 \mathrm{fa}$ of the Dutch Copyright Act, indicated by the "Taverne" license above, please follow below link for the End User Agreement:

www.tue.nl/taverne

Take down policy

If you believe that this document breaches copyright please contact us at:

openaccess@tue.nl

providing details and we will investigate your claim. 


\title{
Clustering-structure Representative Sampling from Graph Streams
}

Jianpeng Zhang, Kaijie Zhu, Yulong Pei, George Fletcher, and Mykola Pechenizkiy

\begin{abstract}
Most existing sampling algorithms on graphs (i.e., network-structured data) focus on sampling from memory-resident static graphs and assume the entire graphs are always available. However, the graphs encountered in modern applications are often too large and/or too dynamic to be processed with limited memory. Furthermore, existing sampling techniques are inadequate for preserving the inherent clustering structure, which is an essential property of complex networks. To tackle these problems, we propose a new sampling algorithm that dynamically maintains a representative sample and is capable of retaining clustering structure in graph streams at any time. Performance of the proposed algorithm is evaluated through empirical experiments using real-world networks. The experimental results have shown that our proposed CPIES algorithm can produce clustering-structure representative samples and outperforms current online sampling algorithms.
\end{abstract}

\section{Introduction}

An increasing number of networks are large-scale and continuously growing in nature. Hence, modeling and analyzing such data in their entirety is becoming infeasible and impractical. One approach to overcome these features of contemporary graph-structured data collections is to sample a representative subgraph and exploit its characteristics. In the existing literature, many sampling methods [3][6][7] focus on sampling from memory-resident static graphs and assume that the sampling algorithms can access the entire graph by multiple passes. However, many contem-

Jianpeng Zhang

Eindhoven University of Technology, 5600 MB Eindhoven, the Netherlands and NDSC, 450002, Zhengzhou, China, e-mail: j. zhang. 4 @tue.nl

Kaijie Zhu

National Digital Switching System Engineering \& Technological R\&D Center (NDSC), 450002, Zhengzhou, China e-mail: kai jiezhu.ndsc@gmail.com

Yulong Pei, George H. L. Fletcher and Mykola Pechenizkiy

Eindhoven University of Technology, 5600 MB Eindhoven, the Netherlands e-mail: $\{y \cdot p e i .1$, g.h.l.fletcher, m.pechenizkiy\} etue.nl 
porary networks can naturally be represented as fully-dynamic graph streams, i.e., nodes or edges are added or removed arbitrarily at any time [10]. To keep pace with such kinds of graphs, we need an incremental sampling method to analyze the interactions within graphs when new entities arrive in a streaming fashion.

Thus there are two research questions which should be addressed. The first challenge is to design an effective and efficient sampling algorithm on fully-dynamic graph streams where edge insertions/deletions are processed in an incremental manner with limited memory. The second challenge is to generate small, yet representative samples of open-ended graph streams. A sampled graph is representative if it preserves selected properties of the original graph. In general, topological properties, such as degree distribution, clustering coefficient distribution and maximum cluster size, are of interest to data scientists. However, existing sampling techniques are inadequate for preserving the inherent clustering structure. To overcome these challenges, we make the following contributions in this paper:

- We propose a Clustering-preserving Partially Induced Edge Sampling (CPIES) algorithm to process the fully-dynamic graph streams. CPIES employs: (i) a clustering-preserving node replacement, (ii) isolated nodes elimination, and (iii) edge-deletion operation. It can retain the inherent clustering structure well, and eliminate the isolated nodes in the sampled counterpart. Moreover, it can handle the edge-deletion requests which is crucial in fully-dynamic setting.

- The empirical experiments on real-world networks show that CPIES is capable of keeping representative/hub nodes rather than peripheral nodes from differen$\mathrm{t}$ clusters in graph streams. It outperforms the state-of-the-art online sampling algorithms in terms of preserving clustering structure.

The remainder of the paper is organized as follows. In Section 2 we provide a brief review of existing work in the areas of graph stream sampling and then give the problem definition in Section 3. In Section 4 the detailed description of the proposed streaming sampling algorithm is given. Experimental evaluation is given in Section 5. The paper is concluded and future work is presented in Section 6.

\section{Related Work}

\subsection{Sampling from Graph Streams}

Graph streams differ from static graphs in three main aspects: (i) the massive volume of edges is far too large to fit into the limited memory; (ii) the topology structure is not fully observable at any point of time (i.e., only sequential access is feasible, not random access); and (iii) efficient, real-time processing is crucial [3]. Motivated by real-world applications, there are some related research on sampling from massive graph streams. Ahmed et al. [1] proposed a generic stream sampling framework for big-graph analytics, called Graph Sample and Hold $(\mathrm{gSH})$. It samples from massive graphs sequentially in a single pass, one edge at a time, while maintaining a small memory footprint. Ahmed et al. [3] extended node sampling, edge sampling and breadth first sampling into streaming setting, and presented a partially-induced edge sampling (PIES) algorithm to sample from graph streams, which maintains 
a sample reservoir of fixed size. A graph priority sampling (GPS) was proposed in [2] for order-based reservoir sampling from massive graph streams. It provides a general way to sample weighted edges according to auxiliary variables so as to accomplish various estimation goals of graph properties. However, it is difficult to use it to sample the inherent clustering structure from fully-dynamic graph streams.

\subsection{Clustering Structure in Graphs}

A cluster in a graph is defined as a set of densely connected nodes that are sparsely connected to nodes outside of the cluster [4]. The discovery of clustering structure in graphs is important as they often correspond to common/latent properties (e.g., interest, role and affiliation) [5]. Although sampling provides a potential solution for inferring and approximating global, latent properties in original graphs, most sampling techniques are not particularly designed to retain the essential property: the inherent clustering structure [12]. To our knowledge, sampling has not previously been applied to the problem of preserving the clustering structure in graph streams.

The work in [6][7] only assessed the degree to which samples are representative of explicit or simple graph properties (e.g., the degree distribution), which can not fully reflect the topology structure. Maiya et al. [8] proposed two sampling algorith$\mathrm{m}$ based on the notion that samples with good expansion properties tend to be more representative of the clustering structure. Newly sampled nodes are chosen either deterministically or probabilistically and the process is continued until we reach the desired subgraph size. However, these approaches are all designed for static graphs to preserve the clustering structure.

\section{Problem Statement}

We focus on the problem of sampling from a fully-dynamic graph stream where edge insertions and deletions are allowed. Formally, for any discrete time-stamp $t \geq 0$, the input is assumed to be a graph $G(t)=[V(t), E(t)]$, presented as a stream of edges $E(t)$ in arbitrary order, where $V(t)$ is a finite set of nodes and $E(t) \subseteq$ $V(t) \times V(t)$ is a set of edges by time $t$. Each edge $e(t)$ is in the form of $\langle u, v, t\rangle$, where $u$ and $v$ are the two incident nodes of the edge and $t$ is the associated timestamp. Initially at time-stamp $t=0$ we have $V(t)=E(t)=\emptyset$, and for any $t>0$, at each discrete time-stamp $t$ we receive a new update $e_{t}=(\bullet,\langle u, v, t\rangle)$ from the edge streams, where $\bullet \in\{+,-\}$. The graph $G(t)=[V(t), E(t)]$ at time-stamp $t$ can be updated as follows:

$$
E(t)= \begin{cases}E(t-1) \cup\langle u, v, t\rangle & \text { if } \bullet=“+" \\ E(t-1) \backslash\langle u, v, t\rangle & \text { if } \bullet=“-"\end{cases}
$$

The aim of graph stream sampling is to generate representative samples, which should obtain a good sample quality, through a single pass on original graph streams. Formally, We denote $\eta(\cdot)$ as any topological graph property, and our objective is to ensure that $G_{s}(t)$ is representative, in which it matches many of the topological properties of $G$, i.e., $\eta(G(t)) \approx \eta\left(G_{s}(t)\right)$. Specifically, in this paper we mainly consider the property of inherent clustering structure. In addition to the sample rep- 
resentativeness requirement, a graph stream sampling algorithm is also required to be computationally efficient (preferably, single-pass) under the limited memory size $\chi$. Formally, we give the problem statement of graph stream sampling. Note that in most cases the sample size of a graph can be defined as the number of nodes in the sample, but it is also feasible to define it as the number of edges in the sample. Here we only consider the former case.

Definition 1. Graph stream sampling: given a graph stream $G(t)$, the sampling algorithm $\mathscr{S}$ aims to produce a sampled graph $G_{S}(t)$ by sampling edges of the graph stream $G(t)$ such that:

- the edges are processed in a sequential order (i.e., not random access) through single pass;

- the memory should satisfy the restriction, i.e., $\left|V_{s}(t)\right| \leq \chi$.

- the topological properties, especially the clustering structure, should be representative and preserved well, i.e., $\eta(G(t)) \approx \eta\left(G_{s}(t)\right)$;

\section{Proposed Sampling Method}

Firstly, we will introduce the state-of-the-art PIES (Partially Induced Edge Sampling) algorithm. Secondly, we will describe our new CPIES algorithm which employs: (i) a clustering-preserving node replacement, (ii) isolated nodes elimination, and (iii) edge-deletion operation to process the fully-dynamic graph streams.

\subsection{The Basic PIES Algorithm}

Basically, PIES [3] is a two-phase sampling algorithm using a single pass for the graph stream: Initially, initial edges in the stream are added to reservoir deterministically in order to accumulate the node reservoir $V_{s}$. After $\left|V_{s}\right|$ reaches the desired sample size $n$, we denote the number of edges in edge reservoir $E_{s}$ by $m$. Then it consists of two phases to process the new edge $e_{t}$ at time $t$ :

- Selection phase: the new edge is added with probability $p=\frac{m}{t}$, where $t$ is the current timestamp. If the probability is not satisfied, the new edge does not sample into the reservoir $V_{s}$. Otherwise, the process goes directly to replacement phase. The rationale is inherited from reservoir sampling and each edge in $E(t)$ has equal probability (i.e., $\frac{m}{t}$ ) of being chosen for the edge reservoir $E_{S}$.

- Replacement phase: If the new edge is selected and at least one incident node has not been sampled into $V_{s}$, the previously sampled nodes in the reservoir $V_{s}$ are replaced based on a certain strategy (namely, Select_Replaced_Node $(\cdot)$ ) in order to maintain the desired size of $V_{s}$.

After these two phases, it adds the new edge to the edge reservoir $E_{s}$ if it$\mathrm{s}$ two incident nodes have already been in the node reservoir $V_{s}$ (i.e., partial induction). After careful analysis, we found that the node replacement strategy Select_Replaced_Node $(\cdot)$ is an alterable module since it allows adopting various strategies to select node that needs to be replaced (i.e., replaceable node). Nesreen proposes two different replacement strategies in [3]. The first strategy selects the replaceable node uniformly at random from the reservoir $V_{s}$, while the second one requires to replace the node kept in $V_{s}$ for the longest amount of time without acquiring more edges, named PIES (Min). However, the current replacement strategies do not consider the clustering structure into account. Thus, we propose a new clustering-preserving replacement strategy to retain the hub nodes in clusters and replace the peripheral nodes to preserve the inherent structure. 


\subsection{Clustering-preserving Node Replacement}

The basic rationale is that the centrality of the nodes in a cluster depends on their degree, and the hub node tend to have higher degree than that of its neighbors. Thus if a node to be replaced is of high degree, we consider that it has higher probability to be the hub node of its cluster such that the sampling process should take further consideration whether it should be selected as the replaceable node.

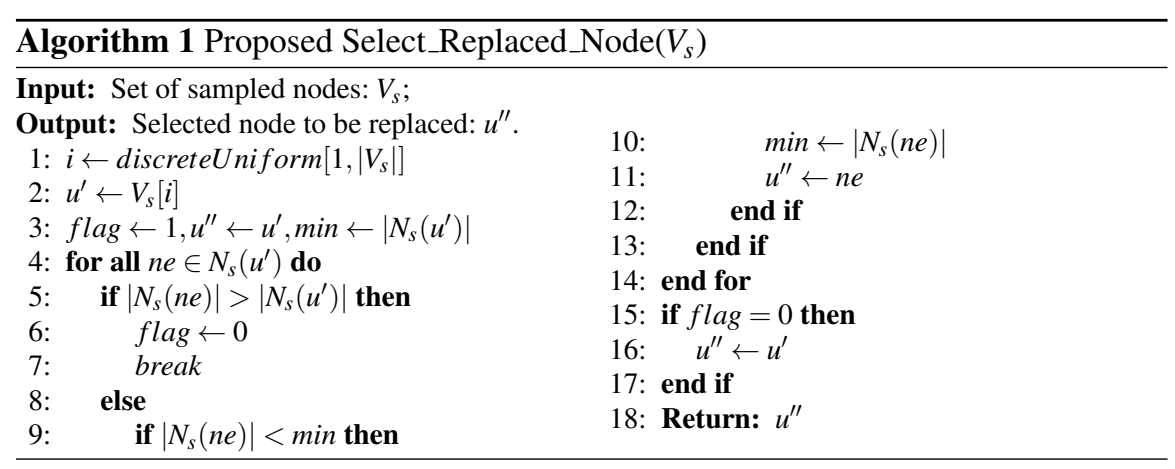

As shown in Algorithm 1, $u^{\prime}$ is the selected node uniformly at random, flag is a boolean variable used to record if the selected node $u^{\prime}$ has higher degree than that of all its neighbors, and $N_{s}\left(u^{\prime}\right)$ is a set of neighbor nodes of $u^{\prime}$ in $V_{s}$. min is used to record the smallest degree of neighbour $N_{s}\left(u^{\prime}\right)$ of node $u^{\prime}$. First, we uniformly select the node $u^{\prime}$ that could be replaced at random. Second, we need to decide whether to replace this node or its neighbour. We compare its degree with that of all its neighbors. If the node's degree is higher than all its neighbors', which indicates the node is probably still a hub node in the cluster, we should not replace the current selected node $u^{\prime}$ but one of its neighbor with the lowest degree as replaceable node instead. In this manner, hub nodes in the sample are kept on the fly, preserving the inherent clustering structure of original graph stream.

\subsection{Isolated Nodes Elimination}

We define sampled nodes in the sample as the node reservoir $V_{s}$, and denote isolated nodes by those nodes kept in $V_{S}$ with no edges attached. The first reason is that in replacement phase of PIES, random selection of the replaceable node may cause the newly added node becomes isolated. Here is a concrete example to illustrate how such kind of isolated nodes are produced.

Example 1: Assuming the new edge $e_{t}=(u, v, t)$ arrives at timestamp $t$ and is going to be inserted into $E_{s}$, when $V_{s}=\left[a_{1}, a_{2}, \ldots a_{n}, u\right]$ (i.e., $u$ belongs to $V_{s}$ but $v$ does not). Since node $v$ does not belong to $V_{s}$, PIES should randomly select a replaceable node for it. However, node $u$ has a probability $p=1 /\left|V_{s}\right|$ to be selected to be the replaceable one so that it will be removed from $V_{s}$ after $v$ is added. Since $u$ does not belong to $V_{s}$ any more after the node replacement, $e_{t}$ will not be inserted such that $v$ becomes an isolated node. 
Obviously, this situation happens from time to time that an incident node of new edge is selected as the one to be replaced. To get rid of this problem, we design a stack structure, named ForbiddenStack and stipulate that nodes existing in this stack should not be selected as replaceable nodes. For each new edge, its associated nodes will be added into ForbiddenStack such that they are forbidden to be chosen as replaceable nodes. When the next edge arrives, nodes in the forbidden stack should be popped up such that it would not influence further node replacement.

The second reason is that after the replaceable node is chosen and needs to be replaced, the neighbours that only connect to the replaceable node become isolated. It is because PIES removes all incident edges of the replaceable node such that the neighbours which only connect with it will have no edge attached. The solution is to check the degrees of neighbours of the replaceable node. If the degrees of neighbors are reduced to zero, those isolated nodes should be removed.

\subsection{Edge-deletion Operation}

Incorporating the ability to delete edges is of crucial importance in a fully-dynamic streaming setting. For example, if the sampling is performed over a sliding window, outdated edges need to be removed from the tail end of the sliding window timely. However, PIES does not take the edge-deletion request into consideration. Thus we propose an efficient deletion method to fully support edge-deletion requests. The pseudocode description is shown in Algorithm 2. Once an edge-deletion is required, if the corresponding edge exists in the sample, it should be directly eliminated from the sample. Otherwise it means the edge was not sampled into the reservoir before and we just ignore it. Note that the edge-deletion request deems to be always satisfied since the deletion of an edge means that the influence of the edge and its incidental nodes on the structure of graph should be eliminated as soon as possible. Moreover, we check the degree of incidental nodes of the deleted edge and remove them if their degree equals to zero after the deletion operation. It guarantees this extension would not introduce extra isolated nodes into the sample. Note that because of the edge deletion operation, the node reservoir may not meet the target size and it will be afterwards compensated again by adding new edges.

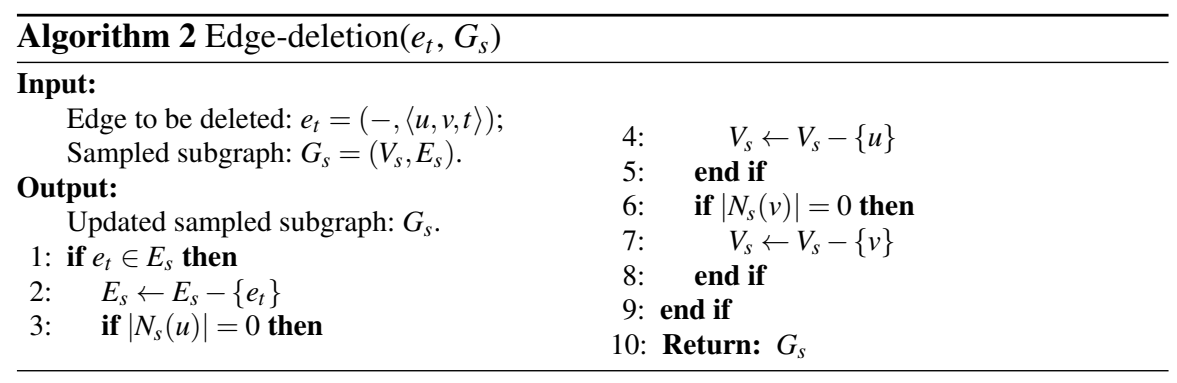




\subsection{Clustering-structure Representative PIES}

Based on these improvements above, we propose the clustering-preserving partiallyinduced-edge-sampling algorithm, namely CPIES, to handle the fully-dynamic graph streams. The algorithmic description is shown in Algorithm 3.

Compared with the basic PIES algorithm, the proposed CPIES is more likely to keep hub nodes from different clusters and avoid isolated nodes such that CPIES should maintain the clustering structure in the samples well.

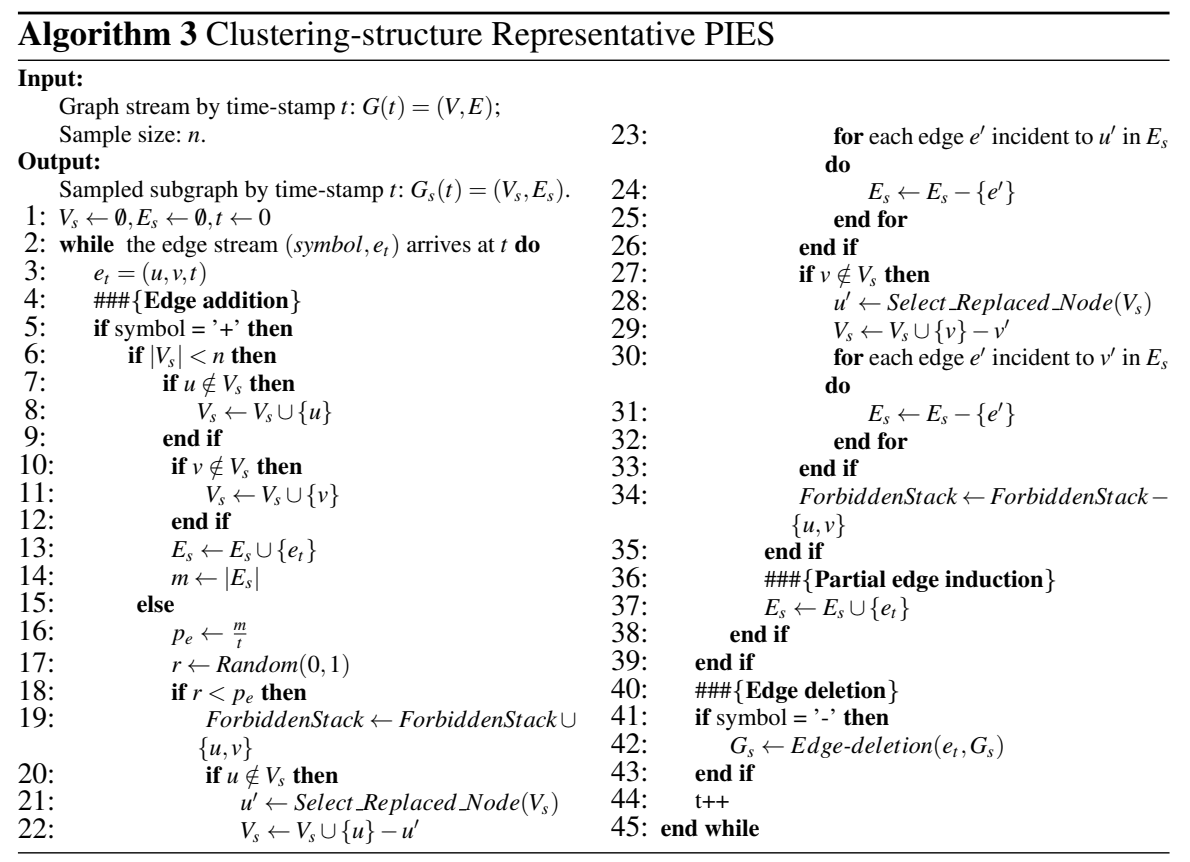

\section{Experimental Evaluation}

This section presents a series of experiments to evaluate the qualities of various sample strategies on their abilities of preserving structural properties. First of all, we will briefly describe the graphs we used and the methodology for the evaluation. Then we discuss the obtained results. We implement streaming edge sampling (StreamES), streaming node sampling (StreamNS), PIES, PIES (min) and proposed CPIES using $\mathrm{C}++$ language. For each sample rate $p$, we experiment with five different runs and calculate various metrics for designated snapshots of real-world networks.

\subsection{Real-world graph streams}

To validate the effectiveness of our proposed method, the real-world graphs are chosen from different domains and they are obtained from the Stanford Large Network 
Dataset Collection [9]. A brief summary of these real networks is shown in Table 1.

Table 1: Summary of real-world graphs used in the experiments. We accumulate graph streams based on specific time interval in order to obtain corresponding snapshots and the statistics of the end streams are given. Abbreviations are described as follows: \# simple edges: number of non-loop and non-duplicate edges; \# snapshots: number of snapshots; \# comps: number of components; $C C$ : the average clusteringcoefficient for all nodes in the graph.

\begin{tabular}{lccccccccccc}
\hline$|G|$ & $|V|$ & $|E|$ & \#simple edges $\begin{array}{c}\text { Time span } \\
\text { (days) }\end{array}$ & $\begin{array}{c}\text { Interval } \\
\text { (months) }\end{array}$ & \#snapshots & \multicolumn{4}{c}{ Statistics of the end streams } \\
\cline { 8 - 12 } & & & & & & \#comps diameter radius density & CC \\
\hline Enron & 151 & 50,572 & 1611 & 1138 & 6 & 7 & 2 & 4 & 3 & 0.14400 & 0.5210 \\
Email-Eu & 986 & 332,334 & 16,064 & 803 & 2 & 14 & 1 & 7 & 4 & 0.03308 & 0.4070 \\
Col_Msg & 1,899 & 59,835 & 13,835 & 193 & 1 & 7 & 4 & 8 & 4 & 0.00772 & 0.1097 \\
Reality & 6,809 & 52,050 & 7697 & 106 & 0.5 & 8 & 1 & 8 & 4 & 0.00033 & 0.0178 \\
Slashdot & 51,068 & 280,443 & 117,340 & 371 & 2 & 7 & 1 & 17 & 9 & 0.00009 & 0.0201 \\
Fackbook & 46,952 & 876,933 & 182,384 & 1560 & 4 & 14 & 842 & 18 & 9 & 0.00019 & 0.1149 \\
\hline
\end{tabular}

\subsection{Evaluation methodology \& measurements}

Current measures of representativeness are inadequate for our target: how well the clustering structure is represented by the counterpart of samples. Hence, we describe our methodology on how to evaluate the clustering structure quantitatively.

Methodology: Firstly, we generate the clusters of each snapshot in original graph streams using any credible clustering algorithm to serve as ground-truth, and then run the same algorithm on the samples generated by these sampling techniques. Note that in order to obtain method-independent results, we utilize two credible and scalable clustering algorithms including: Blondel [4] and BigClam [11] to generate the ground-truth clusters. Secondly, we evaluate the clustering quality of the subgraph generated by each sampling technique using multiple metrics to validate the effectiveness of the methods.

Measurements: We now briefly describe several clustering quality metrics to assess how representative samples are in terms of the inherent clustering structure in the larger network. We consider precision and recall as two main aspects of clustering quality, and each aspect needs to be handled separately without losing the significance of both. Thus, first of all, we utilize $\delta$-precison and $\delta$-recall proposed in [12] to capture the differences of clustering structure between the original graph and the sampled counterpart. $\delta$ is a predefined purity threshold and which measures the correctness of the relation between clusters of original graph and those of sampled counterpart. Two clusters are considered a match if the degree of match is not less than $\delta$. Higher value of $\delta$-precision means that the obtained clusters of $G_{s}$ are more precisely representative of the ground-truth clusters of $G$ while higher value of $\delta$-recall indicates the ground-truth clusters of $G$ are more successfully covered by the obtained clusters of $G_{s}$.

Secondly, we also employ several representative metrics widely used for evaluating clusters in the graph including: adjusted Rand index (ARI) [5], normalized 
mutual information (NMI) [5] and accuracy for number of clusters (ANC) [11] to evaluate the clustering results of the sampled graphs. Note that those metrics are designed solely to assess the clustering quality on the entire graph, not particularly on the sample. To make a fair comparison, we utilize them on the subgraph $G\left(V_{s}\right)$ (i.e., the same set of nodes in the sample).

\subsection{Experimental Results}

Overall performance: In the first experiment, we sample $20 \%$ of the total number of nodes that appear in the stream as it is progressing. In order to evaluate the sample quality in a streaming setting, we take snapshots at different time points in graph streams based on their lifetime (i.e., the number of edges ordered by time-stamps) and compute multiple quality metrics for each snapshot, and then we calculate the "average" scores over all the snapshots to assess the overall performances. We report the performances of these sampling methods for each network in Table 2. Here we only present the results using Blondel clustering, and other results using BigClam algorithms exhibit similar behaviors. Some remarkable conclusions can be drawn as follows:

Table 2: The qualities of various sampling strategies on real graphs $(p=20 \%)$. Besides, all the metrics are calculated by using Blondel clustering algorithms on both original graph and the sampled counterpart. Bold values indicate the best results for corresponding metrics. Please note that the maximum value of $\delta$-recall is equal to the sample rate $p$.

\begin{tabular}{|c|c|c|c|c|c|c|c|c|c|c|c|c|c|}
\hline (a) Enron & Metrics & CPIES & PIES & StreamNS & StreamES & PIES (Min) & (b) Email-Eu & Metrics & CPIES & PIES & StreamNS & StreamES & PIES (Min) \\
\hline \multirow{9}{*}{ Measures } & 1.0-precision & 0.683 & 0.662 & 0.547 & 0.704 & 0.684 & \multirow{9}{*}{ Measures } & 1.0-precision & 0.242 & 0.347 & 0.326 & 0.589 & 0.383 \\
\hline & 1.0-recall & 0.114 & 0.110 & 0.085 & 0.103 & 0.118 & & 1.0-recall & 0.041 & 0.025 & 0.021 & 0.028 & 0.027 \\
\hline & 0.5 -precision & 0.868 & 0.840 & 0.807 & 0.764 & 0.862 & & 0.5 -precision & 0.729 & 0.736 & 0.716 & 0.690 & 0.729 \\
\hline & 0.5-recall & 0.155 & 0.144 & 0.115 & 0.106 & 0.150 & & 0.5 -recall & 0.117 & 0.098 & 0.098 & 0.031 & 0.099 \\
\hline & 0.0 -precision & 0.883 & 0.852 & 0.832 & 0.764 & 0.879 & & 0.0 -precision & 0.785 & 0.774 & 0.770 & 0.708 & 0.773 \\
\hline & 0.0-recall & 0.164 & 0.151 & 0.139 & 0.106 & 0.154 & & 0.0-recall & 0.131 & 0.116 & 0.117 & 0.031 & 0.111 \\
\hline & ANC & 0.861 & 0.857 & 0.821 & 0.548 & 0.825 & & ANC & 0.845 & 0.549 & 0.543 & 0.106 & 0.540 \\
\hline & NMI & 0.784 & 0.766 & 0.751 & 0.736 & 0.770 & & NMI & 0.611 & 0.546 & 0.555 & 0.504 & 0.545 \\
\hline & ARS & 0.646 & 0.606 & 0.561 & 0.343 & 0.595 & & ARS & 0.439 & 0.364 & 0.362 & 0.043 & 0.365 \\
\hline \multirow[t]{2}{*}{ (c) Col_Msg } & Metrics & CPIES & PIES & StreamNS & StreamES & $\overline{P \text { PIES (Min) }}$ & (d) facebook & Metrics & CPIES & PIES & StreamNS & StreamES & IES (Min) \\
\hline & 1.0-precision & 0.397 & 0.149 & 0.120 & 0.307 & 0.192 & \multirow{9}{*}{ Measures } & 1.0-precision & 0.961 & 0.940 & 0.955 & 0.903 & 0.941 \\
\hline \multirow{8}{*}{ Measures } & 1.0-recall & 0.054 & 0.002 & 0.002 & 0.012 & 0.011 & & 1.0-recall & 0.170 & 0.158 & 0.169 & 0.145 & 0.158 \\
\hline & 0.5-precision & 0.455 & 0.222 & 0.210 & 0.478 & 0.281 & & 0.5 -precision & 0.975 & 0.958 & 0.973 & 0.935 & 0.959 \\
\hline & 0.5 -recall & 0.060 & 0.008 & 0.009 & 0.018 & 0.019 & & 0.5 -recall & 0.172 & 0.159 & 0.171 & 0.146 & 0.160 \\
\hline & 0.0 -precision & 0.571 & 0.417 & 0.452 & 0.515 & 0.480 & & 0.0 -precision & 0.980 & 0.963 & 0.978 & 0.940 & 0.963 \\
\hline & 0.0 -recall & 0.093 & 0.046 & 0.048 & 0.023 & 0.054 & & 0.0-recall & 0.172 & 0.160 & 0.172 & 0.146 & 0.161 \\
\hline & ANC & 0.437 & 0.789 & 0.913 & 0.128 & 0.809 & & ANC & 0.951 & 0.928 & 0.856 & 0.795 & 0.931 \\
\hline & NMI & 0.235 & 0.172 & 0.205 & 0.391 & 0.171 & & NMI & 0.781 & 0.759 & 0.779 & 0.742 & 0.763 \\
\hline & ARS & 0.043 & 0.043 & 0.055 & 0.017 & 0.049 & & ARS & 0.699 & 0.666 & 0.686 & 0.597 & 0.669 \\
\hline (e) Slashdot & Metrics & CPIES & PIES & StreamNS & StreamES & PIES (Min) & (f) Reality & Metrics & CPIES & PIES & StreamNS & StreamES & IES (Min) \\
\hline \multirow{9}{*}{ Measures } & 1.0-precision & 0.877 & 0.867 & 0.851 & 0.834 & 0.811 & \multirow{9}{*}{ Measures } & 1.0-precision & 0.514 & 0.498 & 0.549 & 0.485 & 0.471 \\
\hline & 1.0-recall & 0.022 & 0.021 & 0.021 & 0.020 & 0.020 & & 1.0-recall & 0.083 & 0.077 & 0.073 & 0.075 & 0.080 \\
\hline & 0.5 -precision & 0.892 & 0.882 & 0.866 & 0.8 & & & 0.5-precision & 0.925 & 0.835 & 0.832 & 0.852 & 0.797 \\
\hline & 0.5 -recall & 0.027 & 0.027 & 0.028 & 0.6 r & 0.030 & & 0.5 -recall & 0.147 & 0.144 & $\begin{array}{l}\quad 0.134 \\
+\end{array}$ & 0.140 & 0.140 \\
\hline & 0.0 -precision & 0.911 & 0.903 & 0.895 & 0.888 & 0.873 & & 0.0 -precision & 0.861 & 0.868 & 0.892 & 0.880 & 0.842 \\
\hline & 0.0-recall & 0.058 & 0.059 & 0.062 & 0.041 & 0.066 & & 0.0-recall & 0.184 & 0.180 & 0.140 & & 0.184 \\
\hline & ANC & 0.205 & 0.220 & 0.244 & 0.140 & 0.322 & & ANC & 0.886 & 0.897 & 0.652 & 0.869 & 0.792 \\
\hline & NMI & 0.381 & 0.362 & 0.419 & 0.419 & 0.345 & & NMI & 0.885 & 0.879 & 0.892 & 0.860 & 0.876 \\
\hline & ARS & 0.158 & 0.147 & 0.154 & 0.120 & 0.157 & & ARS & 0.728 & 0.714 & 0.748 & 0.620 & 0.705 \\
\hline
\end{tabular}

- The total charts of these results are conclusive. CPIES algorithm outperforms other algorithms in most metrics. This is because that CPIES is biased towards 

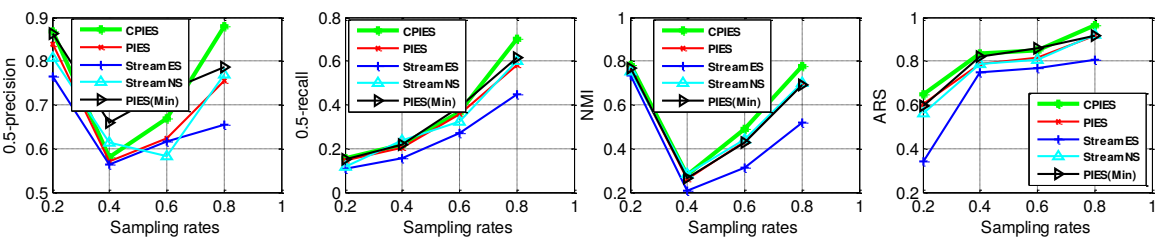

(a) Enron network
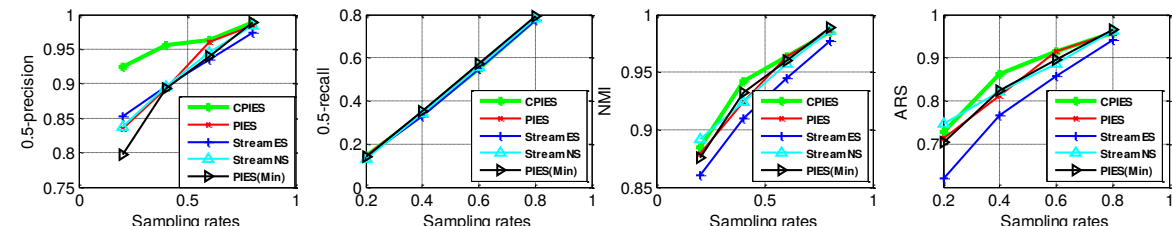

(b) Reality network

Fig. 1: The impact of varying sample rate $p$ on quality metrics on Enron and Reality networks using Blondel clustering.

the central nodes with high degree, and they are good representatives of the underlying clustering structure. For PIES algorithm, the replaced nodes may be the hub nodes of clusters. It will destroy the topology structure and make the clusters in the sample loss of hub nodes and critical connection. Since StreamES and StreamNS sampling uniformly sample the edges/nodes without considering the inherent structure of network, their performances highly depend on the structures of graphs.

- Besides, we found that sampling algorithms that include an induced graph step (i.e., CPIES, PIES and StreamNS) in their process perform better than StreamES (under-sample of edges) in most case because they contain more edges incident to the sampled nodes.

Note that the number of isolated nodes is also analyzed for both CPIES and PIES. Because PIES replace nodes at random, it is inevitable that its sampled counterpart contains some nodes with zero degree (i.e., isolated nodes). Each time a new edge is sampled from the stream, its incident nodes replace randomly selected nodes from the reservoir. This random replacement policy could replace high-degree nodes while isolated nodes remain in the reservoir. However, the proposed CPIES completely avoid isolated nodes and achieves better sample quality.

The impact of sampling rates: In the second experiment, we analyze the impact of the sample rate $p$. The sample rate $p$ controls the ratio of the number of nodes between the original graph and the sampled counterpart. The sample rate $p$ increases gradually from 0.20 to 0.80 with the interval of 0.20 . We run each sampling algorithm 3 trials and take the average of each metrics.

Fig. 1 shows the the average results of various metrics on Enron and Reality networks using Blondel clustering, and we can observe that CPIES performs consistently well with different sampling rates. Note that StreamES does not include an induced graph step so it underestimates the number of edges of the samples such that it fails to preserving the clustering structure well. 


\section{Conclusion}

In this work, we present a new clustering-structure representative sampling method to produce samples from fully-dynamic graph streams. It is capable to retain the influential nodes of clusters and discard isolated nodes such that the clustering structure of original graph is preserved. We empirically demonstrate that CPIES can represent inherent clustering structure of graph streams in an online fashion, and it outperforms current online sampling algorithms in most properties, especially in terms of clustering performance.

In future work, one interesting direction is to explore more general ways to sample the fully-dynamic graph while preserving the clustering structure. Another direction is to design credible measurements to quantitatively evaluate the quality of sample processes. Such quality measures will guide our understanding and study of improved sampling methods.

Acknowledgements This research of Zhang is supported by the Foundation for Innovative Research Groups of the National Natural Science Foundation of China (Grant No. 61521003) and the National key Research and Development Program of China (Grant No. 2016YFB0800101). The research of Pei is supported by the Netherlands Organisation for Scientific Research (NWO).

\section{References}

1. Ahmed, N.K., Duffield, N., Neville, J., Kompella, R.: Graph sample and hold: A framework for big-graph analytics. In: KDD, pp. 1446-1455. ACM (2014)

2. Ahmed, N.K., Duffield, N., Willke, T., Rossi, R.A.: On sampling from massive graph streams. VLDB (2017)

3. Ahmed, N.K., Neville, J., Kompella, R.: Network sampling: From static to streaming graphs. TKDD 8(2), 7 (2014)

4. Blondel, V.D., Guillaume, J.L., Lambiotte, R., Lefebvre, E.: Fast unfolding of communities in large networks. Journal of Statistical Mechanics: Theory and Experiment 10, 008 (2008)

5. Fortunato, S., Hric, D.: Community detection in networks: A user guide. Physics Reports 659, $1-44(2016)$

6. Hübler, C., Kriegel, H.P., Borgwardt, K., Ghahramani, Z.: Metropolis algorithms for representative subgraph sampling. In: ICDM, pp. 283-292. IEEE (2008)

7. Leskovec, J., Faloutsos, C.: Sampling from large graphs. In: KDD, pp. 631-636. ACM (2006)

8. Maiya, A.S., Berger-Wolf, T.Y.: Sampling community structure. In: WWW, pp. 701-710. $\operatorname{ACM}(2010)$

9. Paranjape, A., Benson, A.R., Leskovec, J.: Motifs in temporal networks. In: WSDM, pp. 601-610. ACM (2017)

10. Stefani, L.D., Epasto, A., Riondato, M., Upfal, E.: Trièst: Counting local and global triangles in fully dynamic streams with fixed memory size. TKDD 11(4), 43 (2017)

11. Yang, J., Leskovec, J.: Community-affiliation graph model for overlapping network community detection. In: ICDM, pp. 1170-1175. IEEE (2012)

12. Zhang, J., Pei, Y., H. L. Fletcher, G., Pechenizkiy, M.: Structural measures of clustering quality on graph samples. In: ASONAM, pp. 345-348. IEEE (2016) 